\title{
Metacompreensão e inteligência: um estudo correlacional com estudantes do ensino fundamental
}

\author{
Maria Cristina Rodrigues Azevedo Joly \\ Universidade São Francisco (USF) \\ Anelise Silva Dias
}

\begin{abstract}
Resumo
Este estudo objetivou buscar evidências de validade para a Escala de Metacompreensão (META-FII) por meio da correlação com o WISC-III. Participaram 61 estudantes, 59,0\% do gênero feminino, regularmente matriculadas em uma escola da rede pública de ensino de São Paulo. As idades dos participantes variaram de 10 a 13 anos $(M=11,79 ; D P=1,13)$. A META-FII foi aplicada coletivamente em uma primeira sessão e o WISC-III em duas sessões posteriores, individualmente. Os resultados evidenciaram diferença significativa quanto ao gênero apenas para o QI compreensão verbal. Foram encontradas correlações positivas entre os QIs, verbal, compreensão verbal e total com a META-FII o que atribui a evidência de validade para a escala de metacompreensão.
\end{abstract}

Palavras-chave: metacognição; leitura; avaliação psicométrica.

\begin{abstract}
Metacomprehension and intelligence: a correlate study with elementary students. This study aimed to get validity evidences of Metacomprehension Scale (META-FII) by WISC-III correlation test. The participants were 61 students, 59,0\% were female that were at public elementary school in São Paulo state. The age variation was from 10 through 13 years old $(M=11,79 ; S D=1,13)$. The META-FII scale was collectively administered in the first session and the WISC-III in two post sessions, individually. The results showed significant difference to genre only at verbal comprehension QI. Verbal, verbal comprehension and total QI were positive correlated with META-FII scale which give to the scale validity evidences.
\end{abstract}

Keywords: metacognition; reading; psychometric assessment.

$\mathrm{U}$ $\mathrm{m}$ leitor independente é aquele cuja leitura é caracterizada pela fluência, amplo vocabulário e uso de estratégias que lhe possibilitem compreender melhor as informações obtidas pelos textos (Comissão de Educação e Cultura, 2003). Dentre as características citadas, dar-se-á destaque, no presente estudo, às estratégias de leitura. Isto porque estas requerem a utilização de habilidades que possibilitem a compreensão das informações e são caracterizadas por serem flexíveis, adaptadas às diversas situações que variam de acordo com o texto a ser lido e, por conseguinte, são essenciais à compreensão (Pellegrini, 1996; Serra \& Oller, 2003; Vicentelli, 2000).

As estratégias podem ser consideradas tanto cognitivas quanto metacognitivas. As cognitivas referem-se às ações de domínio de conteúdo e escolha de procedimento que os leitores fazem para atingir os seus objetivos frente à leitura (Bolívar, 2002; Kopke Filho, 2002). Já as metacognitivas relacionamse ao gerenciamento da compreensão que pode vir a ser mais efetiva pelo uso das estratégias adequadas tanto ao tipo quanto à complexidade dos textos (Collins, 1998; Kopke Filho, 2001).

Considerando-se o objetivo do estudo psicométrico em questão, faz-se necessário conceituar metacognição e estratégias metacognitivas de leitura ou metacompreensão. Flavell (1979) definiu metacognição como sendo a cognição sobre a cognição, ou conhecimento e autocontrole da cognição. Assim, a metacognição aplicada à compreensão da leitura pode ser denominada metacompreensão (Block \& Pressley, 2002). Esta permite ao indivíduo planejar, monitorar e avaliar o seu próprio pensamento e processos cognitivos envolvidos durante a leitura, a fim de controlar o conhecimento necessário para viabilizar a compreensão do conteúdo de um texto, objetivando organizá-lo, revisá-lo e modificá-lo (Bolívar, 2002).

As estratégias de metacompreensão podem ser utilizadas antes (planejamento), durante (monitoramento) e após (avaliação) a leitura. No momento anterior à leitura, o leitor procura analisar globalmente o texto e utiliza seu conhecimento prévio sobre o assunto; durante a leitura é feita uma compreensão da mensagem do texto, selecionando as informações de maior relevância, relacionando as mesmas às predições feitas acerca do tema da leitura, a fim de confirmá-las ou refutá-las; e após a leitura, o leitor analisa o conteúdo lido por meio de uma síntese ou releitura do texto, a fim de buscar a relevância das informações do texto, o significado da mensagem, e verificar as diferentes perspectivas apresentadas (Joly, Abreu, \& Piovezan, 2006; Kopke Filho, 1997).

Além dos momentos de leitura, as estratégias de metacompreensāo podem ser classificadas em globais, de suporte 
e solução de problemas. As globais dão uma visão geral do texto ao leitor. Por exemplo, olhar o título, os tópicos e as figuras. As de suporte servem de apoio ao leitor, por exemplo, o uso de materiais de referências, de dicionário e grifos; e as de solução de problemas são utilizadas quando surgem dificuldades na leitura, por exemplo, voltar a ler um trecho do texto (Joly et al., 2006).

Cabe destacar, de acordo com Beltrán (1998), que se as estratégias são utilizadas para identificar diversas competências que têm sido compreendidas como necessárias e úteis para a aprendizagem efetiva, controlar o processamento da informação e retenção da informação, as estratégias metacognitivas estão vinculas à inteligência por dependerem dela para executarem suas funções, por um lado. De outro, por terem flexibilidade para as necessárias adaptações do indivíduo aos contextos e necessidades de aprendizagem a ele impostas, como especificamente no presente estudo que trata das estratégias de metacompreensāo.

Nesse sentido e sob a perspectiva de que as estratégias utilizadas no processo de leitura estão associadas à aferição das habilidades cognitivas, utilizou-se no presente estudo a Escala de Inteligência Wechsler para crianças (WISC-III). Esta foi utilizada para avaliar o perfil cognitivo dos participantes, funcionando assim como variável de critério externo para buscar evidências de validade para a Escala de Metacompreensão (Urbina, 2007). Isso porque a referida escala de inteligência (WISC-III) possibilita, além da avaliação específica da compreensão verbal, a verificação indireta das habilidades de memória, atenção e processamento da informação, habilidades essas envolvidas no processo de compreensão da leitura.

Acrescente-se a isso que as escalas Wechsler, segundo Tulsky e Price (2003), avaliam seis domínios do funcionamento cognitivo, a saber, compreensão verbal, organização perceptual, velocidade de processamento, memória de trabalho, memória auditiva e memória visual. Esses domínios são consistentes com aqueles especificados pelas teorias contemporâneas de inteligência. No mesmo sentido, Zhu e Weiss (2005) consideram que, mesmo as escalas de inteligência Wechsler não tendo sido desenvolvidas de acordo com uma teoria específica de inteligência, foram influenciadas pelos pressupostos de E. L. Thorndike e pelo fator $g$ de C. E. Spearman, consideradas duas teorias clássicas da inteligência.

Faz-se necessário dar relevância aos estudos de correlação feitos entre as escalas de inteligência Wechsler com outros testes de inteligência. Os subtestes da escala Wechsler foram correlacionados com a Full Scale IQ(FSIQ), Differential Ability Scales (DAS), General Cognitive Ability (GCA) e o Kaufman Adolescent and Adult Inteligence Test (KAIT) e os resultados das correlações indicaram boas associações, variando de 0,81 a 0,91 (Zhu \& Weiss, 2005). Tais resultados revelaram consistência das escalas Weschler com outros testes de inteligência e, por conseguinte, pode-se afirmar que seus subtestes avaliam habilidades cognitivas.

No âmbito nacional são poucas as pesquisas que estudam as estratégias metacognitivas, principalmente no que se refere ao seu uso no ensino fundamental (Boruchovit, 2001; Carvalho, 2006; Joly, 2006; Piovezan \& Castro, 2008). Não há registro na base de dados brasileira de referência para a Psicologia, a
BVS-Psi, até o primeiro semestre do ano de 2011, de artigos que descrevam a relação entre metacognição em leitura, metacompreensão e inteligência, quer enquanto procedimentos avaliativos dessas habilidades ou estudos de construção de instrumentos de avaliação. Há registro do estudo de Joly e Istome (2008) o qual buscou evidências de validade para o Teste Cloze Básico - MAR que avalia compreensão em leitura por meio da correlação com WISC III. Foram participantes 52 alunos entre 9 e 15 anos que frequentavam regularmente a $4^{\underline{a}}$ série $(82,7 \%)$ e a $5^{\text {a }}$ série $(17,3 \%)$ do ensino fundamental de uma escola do interior paulista, sendo 53,85\% do gênero feminino. Foi aplicado o Teste de Cloze Básico-Mar coletivamente em uma primeira sessão em sala de aula, e o WISC-III, individualmente em duas sessões posteriores. Os resultados revelaram correlações altas e muito significativas para todos os QI do WISC-III com desempenho em compreensão em leitura, o que é uma evidência de validade para o Teste de Cloze Básico-MAR. Constataram-se diferenças significativas de desempenho em compreensão por gênero, sendo os escores das meninas maiores que dos meninos. A análise de regressão entre as provas possibilitou identificar que QI Compreensão verbal e QI Resistência à distração predizem a compreensão em leitura.

Dada a escassez de investigações nacionais acerca da temática em questão no presente artigo e, visando sustentar, a partir da literatura disponível, os resultados obtidos na investigação que se relata, serão apresentados estudos nacionais e internacionais sobre estratégias metacognitivas de leitura e sobre a relação entre estas e a inteligência.

\section{Estudos sobre estratégias de metacompreensão}

A pesquisa realizada por Boruchovitch (2001) objetivou investigar as estratégias de compreensão de conteúdo durante a leitura para situações de aprendizagem, a saber, em sala de aula, em horários de estudo, em horários de realização de tarefas escolares e em horários de preparação para as provas em casa. Foi solicitado às crianças (estudantes do ensino fundamental) que relatassem como compreendiam as informações lidas nas diferentes situações de aprendizagem investigadas. Os resultados apontaram que a maioria dos participantes buscou apoio social como estratégia para tentar compreender a leitura quando tinha dificuldade. Contudo, a minoria das crianças reportou que, primeiramente, tentava resolver a dificuldade sozinha, por meio da releitura, e quando não conseguia buscava também o apoio social. A alternativa mais citada para solucionar o problema de compreensão foi a de reler. O uso de objetos de apoio, como por exemplo, o dicionário, foi uma categoria de resposta pouco frequente. Relações significativas entre as estratégias de leitura usadas e a repetência foram encontradas, sendo que os alunos repetentes usaram menos estratégias frente a dificuldades de compreensão do que os não repetentes. Não houve, para a amostra avaliada, diferenças estatisticamente significativas entre estratégias de compreensão e idade, gênero e série escolar. Constatou-se a necessidade de que professores e educadores atentem para a importância de se desenvolver um trabalho preventivo para a formação do leitor independente, crítico e reflexivo. 
Carvalho (2006) visou avaliar as estratégias metacognitivas de leitura utilizadas por alunos de $2^{\underline{\underline{a}}}$ a $4^{\underline{\underline{a}}}$ séries do ensino fundamental de escolas públicas e particulares. Foram participantes 451 crianças com idades entre sete e 12 anos $(M$ $=9,50 ; D P=0,97$ ), sendo $53,7 \%$ do gênero masculino, tanto de escolas públicas $(61,2 \%)$, quanto de escolas particulares $(38,8 \%)$ do interior do estado de São Paulo, no Brasil. Para a avaliação das estratégias foi utilizada uma escala de estratégias metacognitivas de leitura para o ensino fundamental, nível 1, aplicada coletivamente em sala de aula, salvaguardados todos os procedimentos éticos para a realização da pesquisa. Os resultados evidenciaram que as crianças da $4^{\mathrm{a}}$ série utilizaram mais estratégias quando comparadas com as das outras séries, para os três fatores da escala, além das maiores médias nos diferentes momentos de leitura. As estratégias mais usadas foram às referentes ao fator solução de problemas. As meninas e as crianças que frequentavam a escola estadual obtiveram maior frequência no uso de estratégias metacognitivas.

Um estudo realizado por Joly (2006) objetivou verificar evidências de validade e precisão para uma escala de estratégias metacognitivas de leitura para crianças na fase inicial de escolarização. Foram sujeitos crianças, que frequentavam regularmente as séries iniciais do ensino fundamental, com idades entre 9 e 14 anos de escolas públicas e particular do interior paulista. Foi aplicada, coletivamente, a Escala de Estratégias de Leitura - nível fundamental I, com 17 itens do tipo Likert, com três pontos (nunca: 0 ponto; algumas vezes: 1 ponto; sempre: 2 pontos), divididas em três categorias, as globais, de apoio e de solução de problemas. Os resultados indicaram que a escala pode ser considerada fidedigna $(\alpha=0,73)$. Também foi realizada uma análise fatorial que revelou a presença de três fatores, estratégias globais, de solução de problemas e de apoio, que explicam $42,12 \%$ da variabilidade da escala. Contudo, foram eliminados quatro itens por possuírem carga fatorial inferior a 0,30 , originando uma nova versão da escala com 13 itens. Diferenças entre a utilização das estratégias, por meio da ANOVA revelou diferença muito significativa para utilização das estratégias de leitura por série; o teste $t$ de Student revelou efeito do gênero para a frequência total de uso da escala de estratégias de leitura, obtendo-se evidências de validade de critério.

O estudo de Piovezan e Castro (2008) objetivou avaliar a compreensão em leitura e o uso de estratégias de leitura em alunos do Ensino Fundamental. A amostra foi composta por 67 alunos da $2^{\underline{a}}$ à $4^{\underline{a}}$ série do Ensino Fundamental de uma escola pública do interior paulista. Do total da amostra, $50,70 \%$ eram do gênero feminino, entre 7 e 13 anos $(M=9,04 ; D P=1,38)$. Utilizou-se um teste Cloze e a Escala de Estratégias Metacognitivas de Leitura. Os alunos mais velhos e de séries mais avançadas apresentaram melhor desempenho na compressão em leitura e menor uso de estratégias de leitura. O desempenho em compreensão em leitura das meninas foi maior, assim como o uso de estratégias de leitura. Em relação às estratégias metacognitivas, os estudantes utilizaram mais as de solução de problemas, no momento durante a leitura; em contrapartida, as estratégias menos indicadas foram referentes ao tipo de estratégia de suporte (durante a leitura) e estratégia global (antes da leitura). Foram encontradas diferenças estatisticamente significativas para o gênero, os participantes do gênero feminino apresentaram médias superiores em relação aos do masculino. Em relação à série, houve diferenças quanto ao fator 1, para as estratégias referentes ao momento durante $\mathrm{e}$ posterior à leitura, bem como para a pontuação total. Quanto às idades, houve diferenças para os fatores 1 e 2 , momentos antes, durante a após, assim como para o total da escala.

Veenman, Wilhelm e Beishuizen (2004) objetivaram determinar em que medida o desenvolvimento de habilidades metacognitivas está associada com inteligência; e, investigar a generalidade versus especificidade do domínio de amadurecimento habilidades metacognitivas. Ambos os objetivos têm implicações importantes para a formação e transferência de habilidades metacognitivas. Participaram quatro grupos etários, sendo três de uma escola na área urbana de Amsterdã (quarta-série $-N=28$; sexta-série $N=28$; e oitava série $-N=30$ ); e um grupo de estudantes universitários $(N=27)$ de uma universidade da Holanda. Os participantes realizaram quatro tarefas de aprendizagem indutiva, representando diferentes domínios. A inteligência, habilidade metacognitiva e desempenho em aprendizagem foram avaliados para cada participante. Os resultados mostram uma habilidade metacognitiva geral, como sendo uma característica relacionada aos grupos etários, em vez de ser de domínio específico. Além disso, as habilidades metacognitivas parecem desenvolver e contribuir para o desempenho em aprendizagem e, parcialmente independente da inteligência. Os resultados revelaram ainda, correlação entre capacidade intelectual, metacognição, aprendizagem e desempenho.

Considerando a relevância do uso de estratégias metacognitivas de leitura para a compreensão das informações (ou seja, as estratégias de metacompreensão); o ensino fundamental como uma etapa de escolarização no qual as crianças iniciam a aprendizagem do uso de estratégias como facilitadoras da compreensão; e, constatando a escassez de instrumentos pertinentes para avaliar estratégias metacognitivas de leitura disponíveis na literatura científica brasileira com qualidades psicométricas consistentes e reconhecidas do ponto de vista científico, objetiva-se com este estudo buscar evidências de validade para a Escala de Metacompreensão para o ensino fundamental (META-FII). Para tanto, realizar-se-á um estudo de correlação entre WISC-III e o META-FII, buscando-se, ainda, analisar a frequência de uso das estratégias de metacompreensão e as habilidades cognitivas dos participantes, além de verificar se as pontuações obtidas na META-FII e no WISC-III diferenciamse em função do gênero, idade e escolaridade dos participantes.

\section{Método}

\section{Participantes}

A amostra foi composta por conveniência, somando-se 61 estudantes, sendo $59,0 \%$ do gênero feminino, regularmente matriculadas em uma escola municipal, da rede pública de ensino do interior paulista, na $5^{\mathrm{a}}(42,6 \%), 6^{\mathrm{a}}(41,0 \%)$ e $7^{\mathrm{a}}$ séries 
$(16,4 \%)$. As idades dos participantes variaram de 10 a 13 anos $(M=11,79 ; D P=1,13)$.

\section{Instrumentos}

Escala de Metacompreensão - META-FII (Joly, 2008). A escala tem o objetivo de avaliar o tipo e frequência de estratégias metacognitivas que as crianças utilizam antes, durante e após a leitura de textos informativos. É composta por 33 itens com respostas do tipo Likert com 3 pontos, a saber: nunca ( 0 ponto), algumas vezes ( 1 pontos) e sempre ( 2 pontos).
Os itens estão divididos em três categorias, que apontam os 3 fatores relacionados às estratégias (suporte, solução de problemas e global). Assim sendo, o fator 1, composto por 15 itens, corresponde às estratégias de apoio à leitura, sendo pois denominado de Estratégias de metacompreensão de suporte à leitura; o fator 2 , conta com nove itens relativos a estratégias usadas para resolver problemas de compreensão e foi denominado de Estratégias de metacompreensão de solução de problemas; e o fator 3, que conta com nove itens, agrupou as estratégias utilizadas para uma análise geral do texto e passou a

\begin{tabular}{|c|c|c|}
\hline Antes de ler um texto, eu costumo: & $\begin{array}{cc}\text { Nunca } & \begin{array}{c}\text { Algumas } \\
\text { Vezes }\end{array}\end{array}$ & Sempre \\
\hline 1. Imaginar como será a história lendo o título. & & \\
\hline \multicolumn{3}{|l|}{ Durante a leitura do texto, eu gosto de: } \\
\hline 10. Opinar sobre a história & & \\
\hline Após a leitura de um texto é comum eu: & & \\
\hline $\begin{array}{l}\text { 29. Reler o texto várias vezes quando tenho dificuldade } \\
\text { para entendê-lo. }\end{array}$ & & \\
\hline
\end{tabular}

Figura 1. Exemplos de itens da META-FII

ser identificado como Estratégias de metacompreensão globais de leitura. A somatória da pontuação pode ser feita por fator, por momento de leitura e considerando-se todos os itens.

A Figura 1 indica três exemplos de item de cada um dos momentos de leitura.

As características psicométricas desse instrumento foram investigadas pelo estudo de validade de construto realizado por Joly (2008) com a META-FII. Participaram 914 estudantes de escolas públicas que frequentavam regularmente de $5^{\text {a }}$ a $8^{\text {a }}$ série do ensino fundamental. Eram 53,8\% do gênero feminino, com média de idade de $13,75(D P=1,1)$. Foi feita uma análise fatorial exploratória para os 33 itens da escala pelo método dos componentes principais com rotação Varimax com normalização Kaiser. Obteve-se índice KMO de 0,89 e o teste de esfericidade de Bartlett revelou adequação da amostra $\left(\chi^{2}[210]=3012,559\right.$; $p<0,001)$. Considerando os valores de comunalidades e carga fatorial por item de, no mínimo, 0,30 , não houve eliminação de item. Definiu-se como a melhor adequação dos itens aquela que contou com três fatores (suporte, solução de problemas e global) cuja variância total explicada foi de $36,0 \%$ e carga fatorial dos itens variou de 0,30 a 0,79 . A precisão foi aferida por meio do Alfa de Cronbach, que indicou uma boa precisão de 0,81 para o total de itens da escala e, considerando cada fator, obteve-se 0,72 para o fator 1 (suporte), 0,62 para o fator 2 (solução de problemas) e 0,46 para o fator 3 (global).

Escala de Inteligência Wechsler para crianças - WISC-III (Figueiredo, 2002). É um instrumento que avalia a capacidade cognitiva de crianças entre 6 e 16 anos e 11 meses. É composto pela Escala Verbal com os subtestes Informação, Semelhanças, Aritmética, Vocabulário, Compreensão e Dígitos e Escala de Execução composta por Completar Figuras, Códigos, Arranjo de Figuras, Cubos, Armar objetos, Procurar Símbolos e Labirintos. Os subtestes Dígitos e Labirinto são suplementares e pelo fato de não serem padronizados para a amostra brasileira e por não serem utilizados diretamente no cálculo dos QIs e dos Índices Fatoriais, não foram utilizados neste estudo. O desempenho da criança nos vários subtestes resulta em três medidas compostas. A somatória ponderada dos subtestes verbais resulta no QI Verbal; a somatória dos subtestes de execução no QI Execução e a somatória dos pontos da Escala Verbal com a de Execução resulta no QI Total. Além desses escores, podem ser calculados quatro escores considerando-se os fatores Compreensão verbal (Informação, Semelhanças, Vocabulário, Compreensão), Organização perceptual (Completar Figuras, Arranjo de Figuras, Cubos, Armar objetos), Resistência à distração (Aritmética e Dígitos) e Velocidade de Processamento (Código, Procurar Símbolos).

Essa versão é brasileira e possui estudos de adaptação, validação, precisão e normatização. Os resultados podem ser observados no manual do instrumento (Figueiredo, 2002) que indica a existência de validade interna do WISC -III, assim como a presença de um fator comum entre os subtestes verbais e para o QI Total, tendo em vista o inter-relacionamento entre os subtestes.

Ainda de acordo com o manual, a partir das diversas análises realizadas para a verificação da qualidade psicométrica do instrumento, destaca-se aqui, os coeficientes de fidedignidade dos subtestes pela idade em anos da amostra. Desta forma, a média dos coeficientes foram adequados, QI verbal $(\alpha=0,95)$, QI execução $(\alpha=0,91)$; QI total $(\alpha=0,96)$; Compreensão Verbal $(\alpha=0,94)$; Organização Perceptual $(\alpha=0,90)$; Resistência à distração $(\alpha=0,87)$; Velocidade de Processamento $(\alpha=0,85)$.

Para a verificação da validade do instrumento, novamente foi destacada a correlação dos escores brutos obtidos no teste com a variável idade, no qual os coeficientes foram adequados, QI verbal $(r=0,82)$, QI execução $(r=0,82)$; QI total $(r=$ $0,84)$; Compreensão Verbal $(r=0,81)$; Organização Perceptual 
$(r=0,79)$; Resistência à distração $(r=0,78)$; Velocidade de Processamento $(r=0,74)$.

\section{Procedimento}

Após a aprovação da pesquisa pelo Comitê de Ética da Universidade São Francisco (protocolo CAAE 0171.142.00008), foi realizado o contato com a escola para a autorização da coleta de dados e agendamento da entrega e recolhimento dos Termos de Consentimento Livre Esclarecido (TCLE) e aplicação dos instrumentos. Após, no primeiro contato com os alunos, foram esclarecidos os objetivos da pesquisa, garantindo ao participante o sigilo e o caráter confidencial da identificação pessoal. Feitos estes esclarecimentos foi solicitado que encaminhassem para os pais ou responsável o TCLE para ser assinado. A amostra foi composta somente por aqueles que entregaram o documento de autorização, iniciando-se então a aplicação dos instrumentos.

Foram necessárias três sessões para a aplicação. A METAFII foi aplicada na primeira sessão, coletivamente, nas próprias salas de aula, o tempo médio de realização foi de 20 minutos. As instruções de realização da escala foram lidas pelo aplicador e as dúvidas que ocorreram foram esclarecidas. Após, os participantes responderam individualmente ao instrumento.

Nas segunda e terceira sessões foram realizadas, de forma individual, a aplicação do WISC-III. Elas ocorreram em dias consecutivos e uma semana após a primeira sessão. Primeiramente foram aplicados os subtestes verbais e depois os de execução. Cada sessão teve uma duração aproximada de 40 minutos. Para a aplicação foram considerados os critérios específicos para cada prova, descritos no manual do teste (Figueiredo, 2002).

\section{Resultados}

Primeiramente apresentam-se os resultados descritivos da META-FII e do WISC-III. Em seguida são descritas as análises estatísticas inferenciais e as correlações total e parcial (controlada por gênero, idade, escolaridade, gênero e idade, gênero, idade e escolaridade) entre os instrumentos. Para a pontuação total na META-FII, destaca-se que poderia variar de 0 a 66 pontos, sendo que a amostra obteve média de 39,51 pontos $(D P=10,66)$, com mínimo de 12 e máximo de 57 pontos. $\mathrm{O}$ resultado revela que os estudantes estão utilizando estratégias de metacompreensão para melhor compreender as informações lidas, uma vez que a média obtida foi superior ao ponto médio da escala.

Foi verificada também a frequência de utilização das estratégias quanto ao momento da leitura, no qual se verificou que as médias foram de 9,86 $(D P=2,94)$ para as usadas antes da leitura, 23,26 $(D P=6,75)$ durante e 5,65 $(D P=2,43)$ para aquelas usadas após a leitura. Contudo, para efeito de comparação entre as médias dos três momentos de leitura, foi realizada uma padronização dos escores, de forma que, a maior utilização de estratégias foi no momento durante a leitura $(M=0,70)$, em relação a antes $(M=0,29)$ e após a leitura $(M=0,17)$. Destaque é dado para os itens 23 (Concentrar-me na leitura quando o texto é difícil), 14 (Observo as figuras da história para entender melhor) e 13 (Ler novamente partes da história quando não entendo) por serem as estratégias mais frequentemente utilizadas, durante a leitura, de acordo com a amostra pesquisada.

No que se refere às pontuações obtidas em cada um dos fatores da escala, verificou-se os seguintes resultados: estratégia global $(M=10,30, D P=3,13)$; suporte $(M=17,69, D P=5,21)$ e solução de problemas $(M=11,59, D P=3,46)$. Novamente, para efeito de comparação entre as médias dos três fatores, foi realizada uma padronização dos escores, de forma que a maior utilização de estratégias foi de suporte $(M=0,54)$, seguida da solução de problemas $(M=0,35)$ e global $(M=0,31)$.

Foram verificadas as estratégias mais utilizadas em relação aos fatores (global, suporte e solução de problemas). A amostra avaliada utilizou mais estratégias de suporte, especificamente o item 14 (Observo as figuras da história para entender melhor) e o item 23 (Concentrar-me na leitura quando o texto é difícil), ambas no momento durante a leitura; e o item 13 (Ler novamente partes da história quando não entendo) de solução de problemas, no momento durante a leitura. As estratégias menos utilizadas por esses alunos foram os itens 5 (Organizar um roteiro para ler), e 20 (Anotar ao lado do texto da história) que são estratégias de suporte, nos momento antes e durante a leitura, respectivamente.

Quanto ao teste WISC-III, também foram verificadas as médias obtidas pelos QIs, a saber: verbal, execução, compreensão verbal, organização perceptual, resistência à distração, velocidade de processamento e total. A média dos estudantes no QI total foi de 106,78 ( $D P=15,57)$. A média mais alta foi alcançada no indicador de QI Execução, com destaque ao fator QI Velocidade de Processamento, conforme pode ser verificado na Tabela 1.

Foram verificadas também possíveis diferenças entre as pontuações obtidas na META-FII e WISC-III em relação ao

Tabela 1

Estatísticas descritivas dos QIs do WISC-III

\begin{tabular}{lcccc}
\hline QI & Média & Desvio Padrão & Mínimo & Máximo \\
\hline Verbal & 99,64 & 23,61 & 14 & 142 \\
Execução & 105,43 & 19,03 & 32 & 131 \\
Total & 106,78 & 15,57 & 61 & 149 \\
Compreensão verbal & 95,36 & 14,51 & 60 & 137 \\
Organização perceptual & 97,83 & 13,02 & 65 & 132 \\
Resistência à distração & 98,47 & 11,63 & 72 & 122 \\
Velocidade de processamento & 100,84 & 11,71 & 74 & 124 \\
\hline
\end{tabular}


gênero e a série da amostra avaliada. Assim, observou-se que para a META-FII, não houve diferença estatisticamente significativa entre os momentos de leitura, bem como entre os fatores, em relação ao gênero.

Da mesma forma, para as medidas de QIs fornecidas pelo WISC-III, não se observaram diferenças significativas para o verbal, execução, total, organização perceptual, resistência à distração e velocidade de processamento. Contudo, somente para o QI compreensão verbal houve diferença significativa $[t(60)=$ $-2,061 ; p=0,044]$ entre as médias obtidas pelos participantes do gênero masculino $(M=99,50)$ em relação aos do feminino $(M=91,94)$ que foi inferior.

Por meio da ANOVA verificou-se a existência de possíveis diferenças de média para os fatores e momento de leitura da META-FII e pontuação nos QIs em função da série. No caso da escala de estratégias não houve diferenças entre os fatores (global, suporte e solução de problemas). Assim como para os momentos antes, durante e após a leitura.

Para os QIs, também não houve diferenças para o verbal, execução, compreensão verbal, organização perceptual, resistência a distração e velocidade de processamento. Entretanto, somente para o QI total verificou-se diferença significativa entre as séries $[F(2,55)=4,66 ; p=0,013]$. O teste de Tukey post hoc (HSD) revelou a formação de dois grupos para explicar a variância do escore total na prova de leitura geral na amostra total. Nesses termos, foram necessários um grupo para os estudantes de $5^{\underline{a}}(M=100,92)$ e $6^{\underline{a}}$ série $(M=109,18)$; e outro para a $6^{\underline{a}}(M=109,18)$ e $7^{\underline{a}}$ série $(M=116,70)$. Constatou-se, assim, que a diferença de médias se deu entre os estudantes de $5^{\mathrm{a}}$ e $7^{\mathrm{a}}$ séries quanto ao desenvolvimento cognitivo.

Para a obtenção de evidência de validade para a EMeL-FII, foi realizada a associação entre os fatores e momentos de leitura com o WISC-III, por meio do teste de correlação de Pearson e encontraram-se associações fracas e significativas entre os momentos antes $(r=0,30 ; p=0,019)$ e durante $(r=0,26 ; p$ $=0,046)$ e fator global $(r=0,32 ; p=0,012)$ com o QI verbal (Tabela 2). Também se observa na Tabela 2 correlações fracas e significativas entre o momento antes da leitura, durante e com os fatores global e solução de problemas com o QI Total.

Verificaram-se ainda associações significativas entre os

Tabela 2

Correlação de Pearson entre os momentos e fatores da META-FII com o WISC-III

\begin{tabular}{|c|c|c|c|c|c|c|c|}
\hline \multirow[b]{2}{*}{ QI } & \multicolumn{4}{|c|}{ Momento } & \multicolumn{3}{|c|}{ Fatores } \\
\hline & & Antes & Durante & Após & Global & Suporte & $\begin{array}{l}\text { Solução de } \\
\text { Problemas }\end{array}$ \\
\hline \multirow{2}{*}{$\begin{array}{l}\text { Verbal } \\
(N=61)\end{array}$} & $r$ & $0,30(*)$ & $0,26(*)$ & 0,22 & $0,32(*)$ & 0,22 & 0,23 \\
\hline & $p$ & 0,019 & 0,046 & 0,083 & 0,012 & 0,092 & 0,070 \\
\hline \multirow[t]{2}{*}{ Execução $(N=61)$} & $r$ & 0,19 & 0,12 & 0,14 & 0,23 & 0,09 & 0,12 \\
\hline & $p$ & 0,146 & 0,379 & 0,273 & 0,076 & 0,484 & 0,367 \\
\hline \multirow{2}{*}{$\begin{array}{l}\text { Total } \\
(N=58)\end{array}$} & $r$ & $0,32(*)$ & $0,36(* *)$ & 0,18 & $0,34(* *)$ & 0,22 & $0,38(* *)$ \\
\hline & $p$ & 0,014 & 0,006 & 0,170 & 0,010 & 0,098 & 0,003 \\
\hline \multirow{2}{*}{$\begin{array}{l}\text { Compreensão verbal } \\
(N=58)\end{array}$} & $r$ & $0,35(* *)$ & $0,38(* *)$ & 0,17 & $0,37(* *)$ & 0,25 & $0,39(* *)$ \\
\hline & $p$ & 0,007 & 0,003 & 0,196 & 0,004 & 0,064 & 0,003 \\
\hline \multirow{2}{*}{$\begin{array}{l}\text { Organização } \\
\text { perceptual } \\
(N=58)\end{array}$} & $r$ & 0,22 & 0,22 & $-0,02$ & $0,28(*)$ & 0,08 & 0,16 \\
\hline & $p$ & 0,092 & 0,103 & 0,867 & 0,035 & 0,565 & 0,225 \\
\hline \multirow{2}{*}{$\begin{array}{l}\text { Resistência à } \\
\text { distração } \\
(N=58)\end{array}$} & $r$ & 0,13 & $0,28(*)$ & 0,20 & 0,19 & 0,12 & $0,31\left(^{*}\right)$ \\
\hline & $p$ & 0,347 & 0,035 & 0,142 & 0,160 & 0,359 & 0,016 \\
\hline \multirow{2}{*}{$\begin{array}{l}\text { Velocidade de } \\
\text { processamento } \\
(N=58)\end{array}$} & $r$ & 0,03 & 0,10 & 0,08 & $-0,01$ & 0,11 & 0,13 \\
\hline & $p$ & 0,821 & 0,471 & 0,533 & 0,920 & 0,422 & 0,328 \\
\hline
\end{tabular}

momentos antes e durante, além dos fatores global e solução de problemas, com o QI compreensão verbal. Além dessas associações, houve também uma correlação fraca entre organização perceptual e o fator global. Pode-se constatar que houve associação fraca entre o momento durante e o fator solução de problemas com o QI resistência à distração (Tabela 2).

Foi realizada uma correlação parcial para verificar possíveis efeitos do gênero na associação entre META-FII e WISC-III. A partir desta análise estatística pode-se perceber que houve associações significativas entre variáveis que na análise anterior não ocorreram, a saber, entre o momento após a leitura e os fatores suporte e solução de problemas com o QI verbal; momento antes e fatores global e solução de problemas com o QI execução. Foi constatada também, associação entre o fator suporte com o QI compreensão verbal. E, pode-se observar que, quando realizada a correlação parcial, a correlação não se mostrou mais significativa entre o QI resistência à distração e durante o momento de leitura (Tabela 3 ). 
Tabela 3

Correlação parcial entre os momentos e fatores da META-FII com o WISC-III controlado por gênero

\begin{tabular}{|c|c|c|c|c|c|c|c|}
\hline \multirow[b]{2}{*}{ QI } & \multicolumn{4}{|c|}{ Momento } & \multicolumn{3}{|c|}{ Fatores } \\
\hline & & Antes & Durante & Após & Global & Suporte & $\begin{array}{l}\text { Solução de } \\
\text { Problemas }\end{array}$ \\
\hline \multirow{2}{*}{$\begin{array}{l}\text { Verbal } \\
(N=61)\end{array}$} & $r$ & 0,40 & 0,47 & 0,26 & 0,45 & 0,28 & 0,49 \\
\hline & $p$ & 0,002 & 0,000 & 0,055 & 0,000 & 0,033 & 0,000 \\
\hline \multirow{2}{*}{$\begin{array}{l}\text { Execução } \\
(N=61)\end{array}$} & $r$ & 0,25 & 0,23 & 0,12 & 0,29 & 0,12 & 0,25 \\
\hline & $p$ & 0,058 & 0,092 & 0,370 & 0,030 & 0,361 & 0,056 \\
\hline \multirow{2}{*}{$\begin{array}{l}\text { Total } \\
(N=58)\end{array}$} & $r$ & 0,33 & 0,37 & 0,18 & 0,34 & 0,22 & 0,39 \\
\hline & $p$ & 0,014 & 0,005 & 0,172 & 0,009 & 0,097 & 0,003 \\
\hline \multirow{2}{*}{$\begin{array}{l}\text { Compreensão } \\
\text { verbal } \\
(N=58)\end{array}$} & $r$ & 0,39 & 0,44 & 0,18 & 0,42 & 0,27 & 0,44 \\
\hline & $p$ & 0,003 & 0,001 & 0,170 & 0,001 & 0,041 & 0,001 \\
\hline \multirow{2}{*}{$\begin{array}{l}\text { Organização } \\
\text { perceptual } \\
(N=58)\end{array}$} & $r$ & 0,24 & 0,25 & $-0,02$ & 0,30 & 0,09 & 0,19 \\
\hline & $p$ & 0,073 & 0,066 & 0,893 & 0,022 & 0,512 & 0,166 \\
\hline \multirow{2}{*}{$\begin{array}{l}\text { Resistência à } \\
\text { distração } \\
(N=58)\end{array}$} & $r$ & 0,10 & 0,24 & 0,19 & 0,15 & 0,10 & 0,28 \\
\hline & $p$ & 0,454 & 0,072 & 0,152 & 0,257 & 0,444 & 0,032 \\
\hline \multirow{2}{*}{$\begin{array}{l}\text { Velocidade de } \\
\text { processamento } \\
(N=58)\end{array}$} & $r$ & 0,01 & 0,06 & 0,08 & $-0,05$ & 0,09 & 0,10 \\
\hline & $p$ & 0,963 & 0,676 & 0,568 & 0,710 & 0,504 & 0,471 \\
\hline
\end{tabular}

Por meio da Tabela 4 observa-se a correlação parcial entre META-FII e WISC-III, com a eliminação de possíveis efeitos da série. A partir desta análise pode-se perceber que houve associações significativas entre variáveis, a saber, entre os fatores suporte e solução de problemas com o QI verbal; momento antes e o fator global com o QI execução. Contudo, não ocorreram algumas das associações significativas com a eliminação da série quando comparadas com as associações a partir da eliminação do gênero, a saber, QI verbal e momento após a leitura; QI execução e fator solução de problemas; e, QI compreensão verbal e fator suporte.

Outra correlação parcial foi realizada de modo a se considerar possíveis efeitos de gênero e série conjuntamente. Os resultados estão apresentados na Tabela 5. Por meio desta análise, pode-se verificar a mudança da magnitude entre algumas variáveis em relação à correlação de Pearson realizada, a saber, QI verbal e momentos antes e durante a leitura, e o fator global da META-FII; e o QI compreensão verbal com os fatores global e solução de problemas. Também, pode-se constatar o surgimento de associações significativas, como o QI execução com o momento antes e com o fator global; QI compreensão verbal com o fator suporte. Contudo, nesta correlação a associação entre o QI resistência à distração com o momento durante a leitura deixou de existir.

\section{Discussão e considerações finais}

O objetivo da presente pesquisa foi buscar evidências de validade para a Escala de Estratégias de Compreensão em Leitura
(EMeL-FII) com a Escala de Inteligência Wechsler para crianças (WISC-III), e verificaram-se também, eventuais diferenças relativas às variáveis, gênero e série.

Os resultados da EMeL-FII demonstraram que os alunos estão utilizando estratégias metacognitivas que favorecem a sua compreensão. A estratégia mais utilizada pela amostra avaliada foi em relação ao momento durante a leitura, que se mostra coerente com os apontamentos de Joly et al. (2006) e Kopke Filho (1997), os quais revelam que no momento durante a leitura, é feita uma compreensão da mensagem do texto, selecionando as informações de maior relevância, relacionando as informações apresentadas com as predições feitas anteriormente, de forma a confirmá-las ou refutá-las. Quanto ao fator, os dados evidenciaram que as estratégias de maior utilização foram de suporte, seguida da solução de problemas. As estratégias de suporte servem de apoio ao leitor, por meio de materiais de referências, como o uso de dicionário e grifos, que contribuem para a melhor compreensão da informação.

Quanto ao teste WISC-III, ressalta-se que a média mais baixa foi a do QI Compreensão Verbal que, no entanto, está compreendida na faixa de desempenho médio previsto, ou seja, na área correspondente a um desvio padrão abaixo da média, considerando que o escore 100 define o desempenho médio da criança em relação à sua faixa etária (Figueiredo, 2002). A média mais alta foi do QI Execução, com destaque ao seu fator QI Velocidade de Processamento. Para Benson (2008) e Primi (2003) a velocidade de processamento e execução é considerada como habilidade cognitiva importante para a leitura. Essas se referem à capacidade de manter a atenção e realizar rapidamente 
Tabela 4

Correlação parcial entre os momentos e fatores da META-FII com o WISC-III controlado por série

\begin{tabular}{|c|c|c|c|c|c|c|c|}
\hline \multirow{2}{*}{ QI } & & \multicolumn{3}{|c|}{ Momento } & \multicolumn{3}{|c|}{ Fatores } \\
\hline & & Antes & Durante & Após & Global & Suporte & $\begin{array}{l}\text { Solução de } \\
\text { Problemas }\end{array}$ \\
\hline \multirow{2}{*}{$\begin{array}{l}\text { Verbal } \\
(N=61)\end{array}$} & $r$ & 0,42 & 0,45 & 0,23 & 0,46 & 0,28 & 0,46 \\
\hline & $p$ & 0,001 & 0,000 & 0,091 & 0,000 & 0,037 & 0,000 \\
\hline \multirow{2}{*}{$\begin{array}{l}\text { Execução } \\
(N=61)\end{array}$} & $r$ & 0,29 & 0,23 & 0,08 & 0,33 & 0,12 & 0,24 \\
\hline & $p$ & 0,029 & 0,084 & 0,565 & 0,014 & 0,363 & 0,070 \\
\hline \multirow{2}{*}{$\begin{array}{l}\text { Total } \\
(N=58)\end{array}$} & $r$ & 0,37 & 0,38 & 0,15 & 0,38 & 0,23 & 0,38 \\
\hline & $p$ & 0,005 & 0,004 & 0,282 & 0,003 & 0,088 & 0,003 \\
\hline \multirow{2}{*}{$\begin{array}{l}\text { Compreensão } \\
\text { verbal } \\
(N=58)\end{array}$} & $r$ & 0,35 & 0,38 & 0,18 & 0,38 & 0,25 & 0,39 \\
\hline & $p$ & 0,007 & 0,003 & 0,185 & 0,005 & 0,065 & 0,003 \\
\hline \multirow{2}{*}{$\begin{array}{l}\text { Organização } \\
\text { perceptual } \\
(N=58)\end{array}$} & $r$ & 0,23 & 0,22 & $-0,03$ & 0,28 & 0,08 & 0,16 \\
\hline & $p$ & 0,090 & 0,107 & 0,822 & 0,034 & 0,575 & 0,239 \\
\hline \multirow{2}{*}{$\begin{array}{l}\text { Resistência à } \\
\text { distração } \\
(N=58)\end{array}$} & $r$ & 0,13 & 0,28 & 0,20 & 0,19 & 0,12 & 0,32 \\
\hline & $p$ & 0,350 & 0,036 & 0,144 & 0,163 & 0,364 & 0,017 \\
\hline \multirow{2}{*}{$\begin{array}{l}\text { Velocidade de } \\
\text { processamento } \\
(N=58)\end{array}$} & $r$ & 0,03 & 0,10 & 0,09 & $-0,02$ & 0,11 & 0,14 \\
\hline & $p$ & 0,840 & 0,469 & 0,490 & 0,901 & 0,419 & 0,313 \\
\hline
\end{tabular}

tarefas simples automatizadas em situações que pressionam o foco de atenção. No estudo realizado por Joly e Istome (2008) a média mais alta foi para o QI Velocidade de Processamento, que pode revelar que os alunos tenham boas condições para processar as informações e relacioná-las aos esquemas mentais existentes, implicando em melhores condições para compreender.

Analisaram-se os resultados quanto às possíveis diferenças entre as pontuações obtidas tanto na EMeL-FII, quanto no WISC-III em relação ao gênero e a série da amostra avaliada. Para a EMeL-FII não se observaram diferenças estatisticamente significativas entre os momentos de leitura, bem como entre os fatores, em relação ao gênero, corroborando o estudo de Boruchovitch (2001). Quanto às possíveis diferenças entre série e as médias para os fatores e momentos de leitura da EMeL-FII, não foram encontradas diferenças entre os fatores e momentos. Contudo, tais resultados foram diferentes em relação aos estudos de Carvalho (2006), Joly (2006), Piovezan e Castro (2008), que revelaram diferenças tanto para série, quanto para o gênero para o uso da escala de estratégias de leitura. Esse fato pode ser decorrência da amostra avaliada pelo presente estudo, que contou com a amostra de apenas uma escola do ensino público, de uma cidade do interior paulista, diferindo dos estudos de Carvalho (2006) e de Joly (2006).

Da mesma forma, para as medidas de QIs fornecidas pelo WISC-III, não se observaram diferenças significativas para gênero e série relativas aos QIs verbal, execução, total, organização perceptual, resistência à distração e velocidade de processamento. Contudo, somente para o QI compreensão verbal houve diferença significativa em relação ao gênero. Quanto à série, verificou-se apenas diferenças para o QI total. No entanto, o estudo realizado por Joly e Istome (2008) demonstrou diferenças entre as idades, série e gênero para o QI total. Tais resultados podem revelar a especificidades do WISC-III quanto aos domínios que avaliam, de acordo com as considerações já feitas por Tulsky e Price (2003).

Para a obtenção de evidência de validade para a EMeLFII, foi realizada a associação entre os fatores e momentos de leitura com o WISC-III. Os resultados mostraram associações entre os QIs, verbal, compreensão verbal e total com a escala de estratégias. Resultados semelhantes foram encontrados no estudo de Veenman, Wilhelm e Beishuizen (2004), indicando uma associação entre a habilidade cognitiva e as estratégias metacognitivas. Tais resultados fornecem evidências de validade para a EMeL-FII, uma vez que se encontrou associações entre os instrumentos. Como mencionado por Beltrán (1998), as estratégias metacognitivas estão associadas à inteligência, uma vez que a habilidade cognitiva auxilia na execução das funções das estratégias, além de possibilitar adaptações do indivíduo aos contextos e necessidades de aprendizagem. Considerando, ainda, possíveis efeitos das variáveis gênero e série, algumas correlações com o QI execução, tornam-se de magnitude fraca. Tais resultados se tornam bastante coerentes com a afirmação de Benson (2008), que destaca a habilidade cognitiva, medida pelo WISC-III, como estando correlacionada com as estratégias de leitura.

A partir dos resultados obtidos, verificaram-se relações entre habilidades cognitivas e habilidades metacognitivas de leitura. Assim sendo, destaca-se aqui a relevância da avaliação das estratégias de metacompreensão, devido a sua importância para o desempenho acadêmico bem sucedido dos estudantes 
Tabela 5

Correlação parcial entre os momentos e fatores da META-FII com o WISC-III controlado por gênero e série

\begin{tabular}{|c|c|c|c|c|c|c|c|}
\hline \multirow[b]{2}{*}{ QI } & \multicolumn{4}{|c|}{ Momento } & \multicolumn{3}{|c|}{ Fatores } \\
\hline & & Antes & Durante & Após & Global & Suporte & $\begin{array}{l}\text { Solução de } \\
\text { Problemas }\end{array}$ \\
\hline \multirow{2}{*}{$\begin{array}{l}\text { Verbal } \\
(N=61)\end{array}$} & $r$ & 0,43 & 0,48 & 0,23 & 0,48 & 0,29 & 0,49 \\
\hline & $p$ & 0,001 & 0,000 & 0,088 & 0,000 & 0,032 & 0,000 \\
\hline \multirow{2}{*}{$\begin{array}{l}\text { Execução } \\
(N=61)\end{array}$} & $r$ & 0,29 & 0,23 & 0,08 & 0,33 & 0,12 & 0,24 \\
\hline & $p$ & 0,030 & 0,083 & 0,569 & 0,014 & 0,368 & 0,070 \\
\hline \multirow{2}{*}{$\begin{array}{l}\text { Total } \\
(N=58)\end{array}$} & $r$ & 0,37 & 0,39 & 0,15 & 0,39 & 0,23 & 0,39 \\
\hline & $p$ & 0,005 & 0,003 & 0,286 & 0,003 & 0,089 & 0,003 \\
\hline \multirow{2}{*}{$\begin{array}{l}\text { Compreensão } \\
\text { verbal } \\
(N=58)\end{array}$} & $r$ & 0,39 & 0,44 & 0,19 & 0,42 & 0,27 & 0,44 \\
\hline & $p$ & 0,003 & 0,001 & 0,157 & 0,001 & 0,042 & 0,001 \\
\hline \multirow{2}{*}{$\begin{array}{l}\text { Organização } \\
\text { perceptual } \\
(N=58)\end{array}$} & $r$ & 0,24 & 0,25 & $-0,03$ & 0,31 & 0,09 & 0,18 \\
\hline & $p$ & 0,072 & 0,069 & 0,851 & 0,022 & 0,522 & 0,178 \\
\hline \multirow{2}{*}{$\begin{array}{l}\text { Resistência à } \\
\text { distração } \\
(N=58)\end{array}$} & $r$ & 0,10 & 0,24 & 0,19 & 0,15 & 0,10 & 0,28 \\
\hline & $p$ & 0,455 & 0,075 & 0,157 & 0,258 & 0,449 & 0,034 \\
\hline \multirow{2}{*}{$\begin{array}{l}\text { Velocidade de } \\
\text { processamento } \\
(N=58)\end{array}$} & $r$ & 0,004 & 0,06 & 0,09 & $-0,05$ & 0,09 & 0,10 \\
\hline & $p$ & 0,979 & 0,671 & 0,526 & 0,697 & 0,499 & 0,452 \\
\hline
\end{tabular}

(Bolívar, 2002; Collins, 1998; Kopke Filho, 2001, 2002). Contudo, cabe destacar algumas limitações do estudo, como o fato dos dados terem sido coletados em apenas uma escola, sendo a mesma pública; e a amostra ser pequena, impossibilitando a generalização dos resultados.

Apesar do número restrito de participantes, as correlações sugeriram evidências de validade para a EMeL-FII com o WISC-III. Isso amplia as evidências de validade para a escala de estratégias metacognitivas colocando-a como uma opção a ser utilizada para avaliar as estratégias de leitura dos alunos do ensino fundamental. Constatou-se também que os QIs verbais, compreensão verbal e de execução são habilidades cognitivas envolvidas no processo de compreensão em leitura, por meio de estratégias de metacompreensão.

Considerando o exposto, sugere-se a realização de novos estudos que foquem principalmente o desenvolvimento de metodologias adequadas que efetivem a utilização das estratégias de leitura no processo educacional visando sua utilização mais frequente, dada sua eficiência constatada. Deve-se levar em conta os tipos de textos peculiares aos diferentes conteúdos acadêmicos como preconizam Pellegrine (1996), Serra e Oller (2003) e Vicentelli (2000).

Destaca-se também, que se deve priorizar estudos que tenham uma perspectiva preventiva e/ou interventiva, visando identificar as variáveis e programas que possibilitem a efetiva utilização da metacompreensão. Nesse sentido, poder-se-á subsidiar professores a fim destes implementarem atividades que envolvam o ensino de estratégias de metacompreensão.

\section{Referências}

Beltrán, J. A. (1998). Processos, estrategias y técnicas de aprendizaje. Madri: Síntesis.

Benson, N. (2008). Cattell horn carroll cognitive abilities and reading achievement. Journal of Psychoeducational Assessment, 26, 27-41.

Block, C. C., \& Pressley, M. (2002). Introduction. In C. C. Block \& M. Pressley (Orgs.), Comprehension instruction: Research-based best practices (pp. 1-7). Nova Iorque: Guilford Press.Bolívar, C. R. (2002). Mediación de estratégias metacognitivas en Tareas Divergentes Y Transferencia Recíproca. Investigación Y Postgrado, 17(2), 1-20.

Boruchovitch, E. (2001). Aprender a aprender: propostas de intervenção em estratégias de aprendizagem. Educação Temática Digital, 8(2), 156-167.

Carvalho, M. R. (2006). Estratégias metacognitivas de leitura utilizadas de $2^{\underline{a}}$ a $4^{\underline{a}}$ série do Ensino Fundamental. (Dissertação de Mestrado não publicada), Universidade São Francisco, São Paulo.

Collins, M. (1998). Young children's reading estrategies. Australian Journal of Language and Literacy, 2(1), 55-64.

Comissão de Educação e Cultura - Câmara dos Deputados. (2003). Grupo de trabalho - alfabetização infantil: os novos caminhos - relatório final. Brasília: Estação Gráfica.

Figueiredo, V. L. M. (2002). Escala de inteligência Wechsler para crianças. Manual. Adaptação e padronização de uma amostra brasileira. São Paulo: Casa do Psicólogo.

Flavell, J. H. (1979). Metacognition and cognitive monitoring: a new area of cognitive developmental inquiry. American Psychologist, 34(10), 906-911.

Joly, M. C. R. A. (2006). Escala de estratégias de leitura para etapa inicial do ensino fundamental. Estudos de Psicologia, 23(3), 271-278.

Joly, M. C. R. A. (2008). Escala de metacompreensão - Ensino Fundamental II(META-FII). Relatório de Pesquisa, Universidade São Francisco, Itatiba. 
Joly, M. C. R. A., \& Istome, A. C. (2008). Compreensão em leitura e capacidade cognitiva: estudo de validade do teste Cloze Básico - MAR. Psic, 9(2), 219-228.

Joly, M. C. R. A., Abreu, M. C., \& Piovezan, N. M. (2006). Validação concorrente de uma escala metacognitiva de leitura para universitários brasileiros. Psicologia, Educação e Cultura, 10(2), 447-460.

Kopke Filho, H. (1997). Estratégias para desenvolver a metacognição e a compreensão de textos teóricos na universidade. Psicologia Escolar e Educacional, 1(2-3), 59-67.

Kopke Filho, H. (2001). Estratégias em compreensão da leitura: conhecimento e uso por professores de língua portuguesa. (Tese de Doutorado).Universidade de São Paulo, São Paulo.

Kopke Filho, H. (2002). Repertório de estratégias de compreensão da leitura e conhecimentos metacognitivo de professores de Língua Portuguesa. Psicologia Escolar e Educacional, 6(1), 67-80.

Pellegrini, M. C. K. (1996). Avaliação dos níveis de compreensão e atitudes frente à leitura em universitários. (Dissertação de Mestrado não publicada), Universidade São Francisco, Paulista.

Piovezan, N. M., \& Castro, N. R. (2008). Compreensão e estratégias de leitura no ensino fundamental. Psic: revista da Vetor Editora, 9(1), 53-62.

Primi, R. (2003). Inteligência: avanços nos modelos teóricos e nos instrumentos de medida. Avaliação Psicológica, 1, 67-77.

Serra, J., \& Oller, C. (2003). Estratégias de leitura e compreensão do texto no ensino fundamental e médio. In A. Teberosky (Org.), Compreensão de leitura: a língua como procedimento (pp. 35-44). Porto Alegre: Artmed.

Tulsky, D. S., \& Price, L. R. (2003). The joint WAIS-III and WMS-III factor structure: development and cross-validation of a six-factor model of cognitive functioning. Psychological Assessment, 15(2), 149-162.

Urbina, S. (2007). Fundamentos da testagem psicológica. Porto Alegre: Artmed.

Veenman, M. V. J., Wilhelm, P., \& Beishuizen. J. J. (2004). The relation between intellectual and metacognitive skills from a developmental perspective. Learning and Instruction, 14(1), 89-109.

Vicentelli, H. (2000). Problemática de la lectura en estudiantes universitarios. Psicologia Escolar e Educacional, 3, 195-202.

Zhu, J., \& Weiss, L. (2005). The Wechsler Scales. In D. P. Flanagan, J. L. Genshaft \& P. L. Harrison (Orgs.), Contemporary intellectual assessment: theories, tests and issues (2 ${ }^{\mathrm{a}}$ ed., pp. 297-324). Nova Iorque: Guilford.

Maria Cristina Rodrigues Azevedo Joly, doutora em Psicologia Escolar e do Desenvolvimento Humano pela Universidade de São Paulo e pós-doutora em Avaliação Psicológica pela Universidade do Minho, é professora Associada na Universidade São Francisco. Endereço para correspondência: Rua Alexandre Rodrigues Barboisa, 45 13251-900 Itatiba, SP. Telefones: (19) 81377493-cel/ (19) 38693328-resid/ (19)32334233-FAX/ (11) 45348103-USF. E-mail: cristina.joly@usf.edu.br

Anelise Silva Dias, doutora em Psicologia pela Universidade São Francisco. E-mail: anelise_dias@yahoo. com.br 